\title{
Anorectal anomalies: with an international proposed classification
}

\author{
H. H. NIXON \\ F.R.C.S.
}

Hospital for Sick Children, Great Ormond Street, London, W.C.1

ANORECTAL anomalies occur in about 1 in 3000 births. The traditional name of imperforate anus is unfashionable because there is usually an abnormal opening present. Persistence of the anal membrane which is so commonly mentioned in embryological texts is extremely rare in practice, although stenosis at this site is a little less uncommon $(1.6 \%$ in a consecutive series of 300 ). Observation of surgical anatomy and of comparative anatomy (e.g. Keith, 1908) suggest that failure of completion of the division of the cloaca into urinary and intestinal parts is a common primary error. Associated with this the anlage of the proctodeum fails to develop to its normal anatomy. Another factor is the 'covering' of a potentially adequate anus by overfusion of the lateral genital folds or their posteriorly related anal tubercles (Stephens, 1963; Browne, 1951; Gans \& Friedman, 1961; Bill \& Johnson, 1958). Hence, the abnormal opening or potential opening may be looked on as an imperfect or primitive anusalthough many prefer to call it a fistula when it is so imperfect that it cannot be used by simple enlargement (Scott, 1966; Kiesewetter \& Nixon, 1967).

Three main groups can be recognized. In rectal agenesis the bowel ends above the pelvic floor, typically with a fistula to the prostatic urethra in the male or to the posterior vaginal fornix in the female (Fig. 1). About $40 \%$ of anorectal anomalies are of this type. In the second group the bowel traverses the pelvic floor with its crucial puborectalis sling but fails to migrate back to the normal anal site. Hence, a vestibular vulval or perineal opening ('ectopic anus' or fistula) exists. Like all ectopic openings these tend to be stenotic. The third main group is the 'covered' anus. An epithelial excrescence may cover the opening at the normal anal site or the orifice may be projected forwards onto the perineum or vulva in the female. In the male the track may extend forward on to the scrotum or even the penile raphe. A thin membranous area usually exists in part of the track through which dark meconium can be seen and this may perforate spontaneously (Fig. 2). (This thin area when present over the normal anal site was misinterpreted as a persistent anal mem-

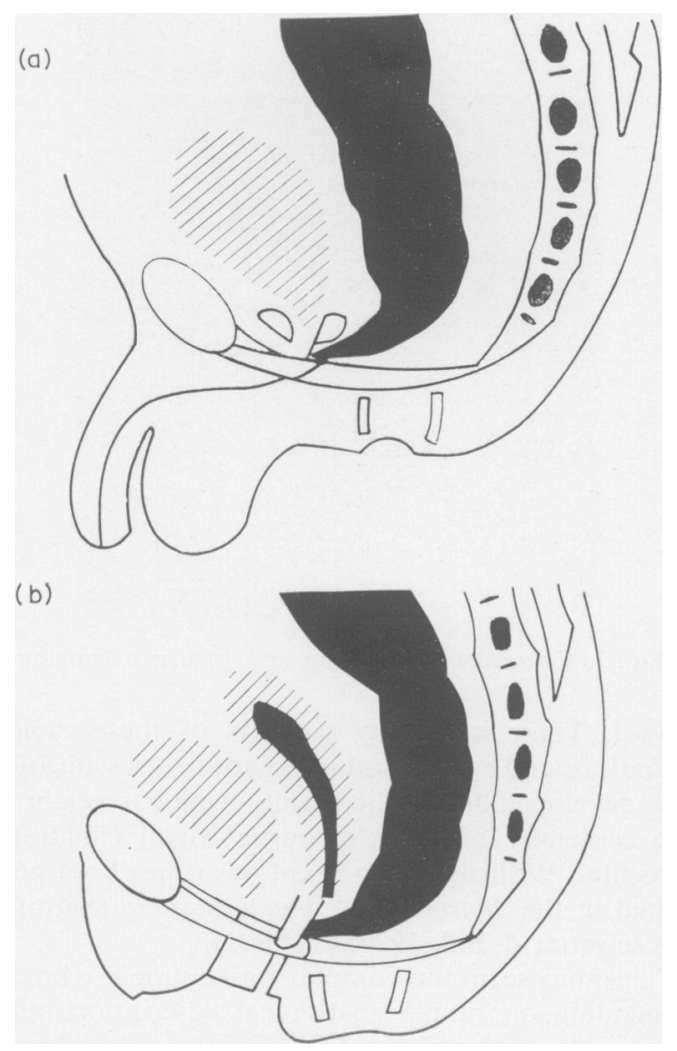

Fig. 1. (a) Rectal agenesis with recto-urethral fistula. (b) Rectal agenesis with rectovaginal fistula (after Santulli, Kiesewetter \& Bill, 1970).

brane in the past, but it is at the lower end of the canal and not at the junction of proctodeum and endoderm.)

The most vital distinction between the different types from a practical point of view is that anorectal agenesis leaves the bowel above the levator floor whereas in the ectopic anus and covered anus types the bowel traverses the levator before its abnormal termination. Hence, in these latter the puborectalis sling, the most important striated muscle involved in continence, retains a correct relationship to the 


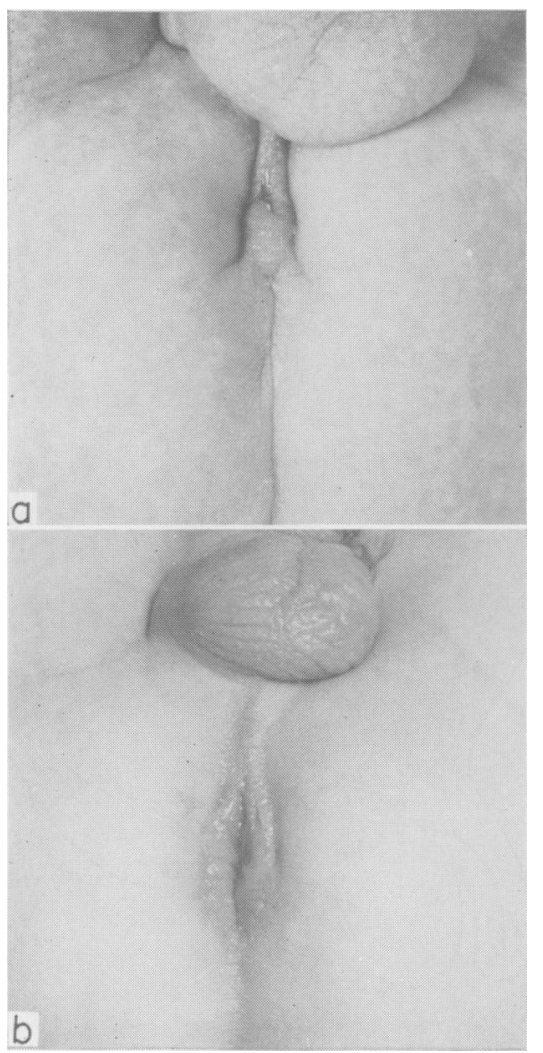

FIG. 2. Covered anus (a) before and (b) after uncapping.

bowel. There are many variants of these typical situations and a proposed international classification was developed during the recent meeting to celebrate the centenary of the Melbourne Royal Children's Hospital. With slight modifications it has been published in the Journal of Pediatric Surgery (Santulli, Kiesewetter \& Bill, 1970) (Table 1).

This may seem too complex for routine use but by depending on simple anatomical description minimizing the embryological implications, it is hoped that this will produce a classification which can be used for the comparison of results obtained with different methods in different centres. The old Ladd \& Gross classification is very imperfect in this respect because the majority of patients come into their type III which includes some abnormalities which can be treated by minor surgery to achieve normal continence and others in which even major surgery can only be expected to produce adequate learned continence when the child is old enough to co-operate. The classification is not in fact as complicated as it looks. It makes the same vital distinction between high and low (supralevator or translevator) abnormalities and allows for the fistulae being open or merely fibrous attachments without lumen, for variations in the level of the terminal opening, and whether or not the bowel retains a normal width down to this level or becomes narrow some distance above it. These variations can have significant effect on the ease and manner of treatment.

\section{Diagnosis}

The most important step in diagnosis is a careful inspection of the baby and of the perineum. In the low abnormalities the abnormal orifice of the ectopic opening can be seen on the perineum or in the vulva or vestibule. In the covered anus the epithelial excrescence can be recognized as a median bar across the anus or as a track running forward from that site and including pearly epithelial collections, and in the thin area the dark green meconium will be seen through the membrane. In the high abnormalities there will be no visible orifice. However, in the female meconium may be seen to come from the vagina and in the male the first urine passed may be noticeably stained with meconium. (Microscopic examination of the first urine may also show squames confirming the presence of a fistula to the urinary tract.) Rectal examination will reveal the less common anorectal stenosis as a cause of delayed passage of meconium or of abdominal distension.

Palpation may reveal a short sacrum. This is important because a sacrum of three segments or less will assuredly be associated with a neurological deficit in the pelvic floor affecting the prognosis for urinary control as well as that of the bowel. In the general examination of the baby for associated abnormalities signs of congenital heart disease or of oesophageal atresia are particularly to be borne in mind. Since some minor or major urinary tract anomaly will be associated in a quarter of the cases of low anorectal anomaly and over one third of the high anorectal anomalies a pyelogram and voiding cystogram should be performed in all cases at a suitable time in their management.

It is traditional to make an 'invertogram'. This is a plain radiograph taken after the baby has been inverted for a reasonable period to allow the gas to outline the lower end of the bowel. This cannot be achieved reliably during the first $24 \mathrm{hr}$. Stephens' modification in which a lateral view is taken centred on the greater trochanter is of more value than the classical Wangensteen \& Rice (1930) A.P. view. The custom of placing a marker on the normal anal site is not helpful because the distance to this point depends on the distribution of the ischiorectal and peri-anal fat more than anything else. Stephens' pubococcygeal line relates the gas bubble to a skeletal marker and is of more significance. The level of the puborectalis sling is just below this line. A line at the junction of the body and tail of the comma- 
TABLE 1 .

\begin{tabular}{|c|c|}
\hline Male & Female \\
\hline $\begin{array}{l}\text { High (supralevator) } \\
\text { (1) Anorectal agenesis } \\
\text { (a) Without fistula } \\
\text { (b) With fistula } \\
\text { (i) Rectovesical } \\
\text { (ii) Rectourethral } \\
\text { (2) Rectal atresia }\end{array}$ & $\begin{array}{l}\text { (a) Without fistula } \\
\text { (b) With fistula } \\
\text { (i) Rectovesical } \\
\text { (ii) Rectocloacal } \\
\text { (iii) Rectovaginal-high }\end{array}$ \\
\hline $\begin{array}{l}\text { Intermediate } \\
\text { (1) Anal agenesis } \\
\text { (a) Without fistula } \\
\text { (b) With fistula } \\
\text { (i) Rectobulbar } \\
\text { (2) Anorectal stenosis }\end{array}$ & $\begin{array}{l}\text { (a) Without fistula } \\
\text { (b) With fistula } \\
\text { (i) Rectovaginal-low } \\
\text { (ii) Rectovestibular }\end{array}$ \\
\hline $\begin{array}{l}\text { Low (translevator) } \\
\text { (1) At normal anal site }\end{array}$ & $\begin{array}{l}\text { (i) Covered anus-complete } \\
\text { ii) Anal stenosis }\end{array}$ \\
\hline 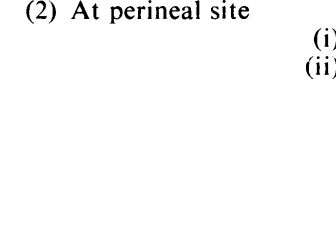 & $\begin{array}{l}\text { i) Anterior perineal anus } \\
\text { i) Anocutaneous fistula } \\
\text { (covered anus-incomplete) } \\
\text { (3) At vulvar site } \\
\text { (i) Vulvar anus } \\
\text { (ii) Anovulvar fistula } \\
\text { (iii) Anovestibular fistula }\end{array}$ \\
\hline Miscellaneous & \\
\hline
\end{tabular}

shaped ischial shadow is an alternative skeletal marker for the pelvic floor (Kelly, 1969) (Fig. 3). But the low abnormalities can be recognized by clinical examination and the exact nature of the high anomalies in modern practice are worked out by study of the 'distal loopogram' performed after transverse colostomy and related to the voiding cystogram. Probably the most helpful observation on the invertogram is its demonstration of the normality or otherwise of the development of the sacrum.

\section{Treatment}

Ectopic anus (types: anterior perineal anus: vulvar anus: anovulvar fistula: anovestibular fistula)

The simple cut-back described by Denis Browne is all that is needed to make the imperfect anus large enough to work where it lies (Fig. 4). It is, however, important to follow this with daily dilatations for 3 months until the wound is not only healed but supple

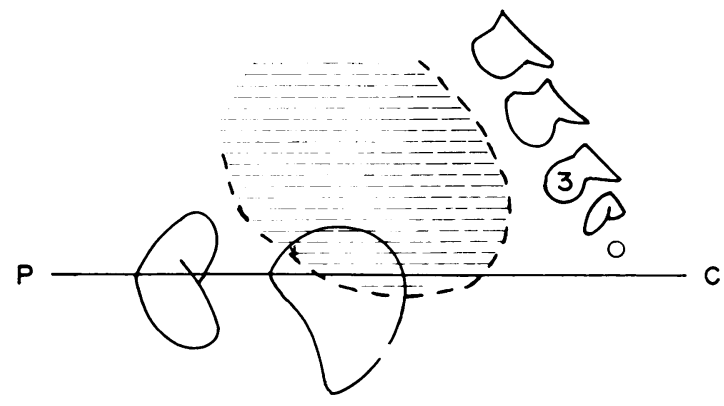

FIG. 3. 'The invertogram', showing the $\mathrm{P}-\mathrm{C}$ line from the body of the pubis to the estimated position of the coccyx. The line also passes through the shadow of the ischium at the junction of its upper quarter (crescent) with the lower three quarters (tail).

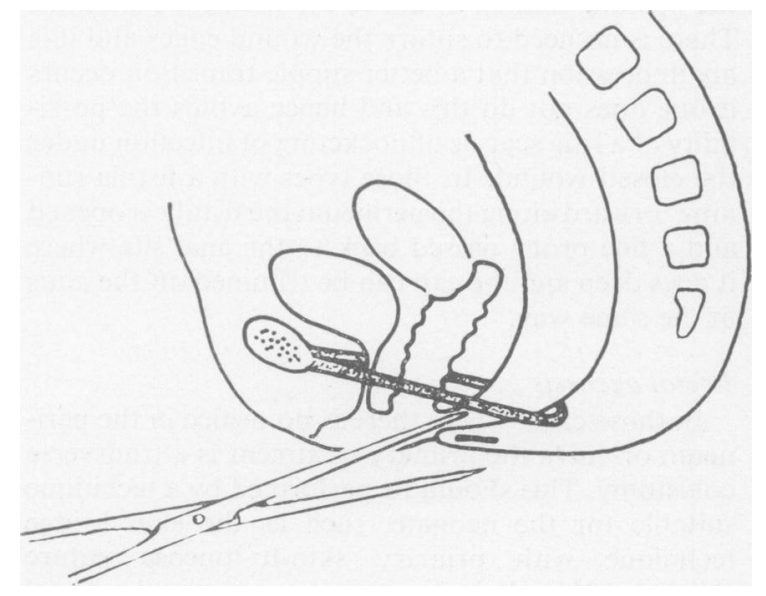

FIG. 4. The cutback procedure for covered anus. 
again. In a newborn size 12 or 13 Hegar is an adequate size and when mother is continuing this at home her fifth finger will usually be of suitable size and safer to use because she can feel what is happening as she inserts it. Intravenous pyelography and voiding cystography are carried out before the child leaves hospital. These children will often appear well and pass the soft stools of milk-feeding and particularly breast-milk feeding without apparent trouble. It is most unwise to delay in these circumstances because considerable rectal distension can take place before the child is obviously distressed on defaecation and the resultant inertia can give rise to chronic constipation and overflow-incontinence which requires prolonged and unpleasant treatment for the child. Furthermore, the required dilatations leave no psychological aftermath when they are carried out in the first 3 months of life but delay to a later period can be associated with much stress for the child and interfere with the normal development of bowel habit at the usual time. The use of simple dilatations without a preliminary cut-back is also rarely adequate except in cases of perineal ectopic anus not far in front of the normal anal site. One disadvantage is that the orifice remains so far anteriorly that there is a tendency for the bowel to drive the faeces down behind it against the pelvic floor, producing incomplete evacuation and soiling. Also, in those cases where the vestibular ectopic anus is immediately adjacent to the hymen the anterior half of the orifice is more easily dilated than the posterior so that the effect of the dilatation is to stretch the opening up into the vagina unless the posterior wall is first cut back.

\section{Covered anus}

In the type at the normal anal site (median band of the anus) the epithelial bar is simply excised and the opening dilated as above for the next 3 months. There is no need to suture the wound edges and it is my impression that a better supple transition occurs if one does not do this and hence avoids the possibility of a ring scar or of pocketing of infection under the closed wound. In those types with a fistula running forward along the perineum the fistula is opened and a fine probe passed back to the anal site where it goes deep and the cap can be trimmed off the anus in the same way.

\section{Rectal agenesis}

In those cases where there is no orifice in the perineum or vulva the primary treatment is a transverse colostomy. This should be performed by a technique suitable for the neonate, such as the skin bridge technique with primary skin-to-mucosa suture (Nixon, 1966). It is important to empty the distal loop at operation to avoid contamination of the urinary tract through the fistula. A few days later intravenous pyelography and voiding cystography and distal loop contrast examination will elucidate the exact nature of the anomaly. The distal loop should be washed out with saline fortnightly until definitive operation to avoid the collection of inspissated mucus or overspill of faeces. It is usual to delay the definitive operation until the child is 9 months old because the essential of any operation for this abnormality is to get the bowel through the levator floor in front of the puborectalis sling without damaging that sling. The continence results of neonatal abdomino-anal pullthrough operations suggest that the dilatation necessary to make the track through the pelvic floor is in the neonatal period relatively too great to leave the levator competent to achieve an adequate shut-off at the anorectal junction. At present we prefer an operation in which a preliminary sacral approach, as described by Stephens, is used to visualize the puborectalis sling and mark the track for the bowel in front of it. The patient is then turned on his back and the bowel brought down to the anal site by coring out the mucosa of the rectum and drawing the colon through this tunnel as described by Rehbein (Kiesewetter, 1967). The object of this procedure is to be sure of getting in front of the puborectalis sling with the minimal chance of damaging this, and to avoid the need to dissect around the bladder neck and prostatic urethra where the pelvic splanchic nerve plexuses fall together in the midline in the absence of a normal rectum (Scott, 1959). I add to this a trapezoid excision of skin in front and behind the newly formed anus so as to invert this and reduce the tendency to prolapse of mucosa (and perhaps to get the sensitive anal skin far enough inside the canal to be of some value as a warning of impending defaecation).

In this group one does not expect normal continence to be achieved at the normal time as in the low anomalies. It is usually necessary to teach the child to make regular efforts at defaecation rather than expecting a normal 'call to stool'. There is a tendency to soiling rather than involuntary defaecation in a considerable proportion as a result of incomplete evacuation of the rectum leading to rectal inertia with loss of filling sensation and overflow incontinence. Others may soil as the result of the low resting pressure in the anal canal. The symptoms tend to improve as the child gets older and is more understanding of the need for continence. Regular aperients or enemas may be needed to stimulate effective evacuation and hence reduce soiling. For a few a colostomy may still be needed as a last resort. The problems of school life and particularly adolescence with a colostomy justify the use of secondary operations to attempt to improve 'sphincter' function before this decision is made. 


\section{Anorectal stenosis}

This less common type is mentioned because its management may not be as simple as it appears. In about half the cases the stenosis is a string-like one at the junction of proctodeum and endodermal lining of the upper part of the anatomical anal canal. It responds simply to dilatation. But in about half the patients there is a more widespread fibrosis which involves the internal sphincter. When the anal canal is dilated sufficiently to prevent it causing resistance to the passage of stool then the anal canal may be too inelastic to close easily and completely so that soiling can be a problem requiring further management.

In the perineal and vulval ectopic anus the simple cut-back gives rise to what Denis Browne called the 'shot-gun perineum' with both barrels alongside but functioning normally. No further treatment is required and an experience over many years with some patients having reached married life confirms that it is a fully acceptable situation. However, in cases of the vestibular ectopic anus where the orifice is immediately adjacent to the hymen and the bowel extends more directly up behind the vagina rather than going backwards for a considerable distance as in the vulval ectopic anus, the final anal orifice, although satisfactorily continent may be awkwardly placed for hygiene. In these cases a perineal transplantation of the anus to the normal site can be carried out more satisfactorily in the older child who has already achieved continence and who understands the object of the operation and will cooperate in aftercare. It does not seem necessary to consider primary anal transplant in these cases when the delicate tissues make the operation more prone to complications.

\section{Results}

Dr G. Willital of Erlangen has recently reviewed the follow-up records of 300 consecutive patients referred to my unit for anorectal anomalies. These will be published in more detail elsewhere. In the low abnormalities normal continence was achieved in $93 \%$ of cases (ectopic $90 \%$ and covered anus $96 \%$ ). In the high abnormalities (rectal agenesis) those in which the puborectalis sling was viewed at operation achieved a satisfactory stage of learned control in $92 \%$ of cases, but only in $42 \%$ did this approach normal continence. In those earlier cases where the sling was not visualized the corresponding figures are only $34 \%$ and $15 \%$ respectively.

Only 171 of the 300 patients were referred to the clinic before a previous attempt had been made to construct the anus. In those cases of rectal agenesis referred for secondary treatment $80 \%$ achieved 'satisfactory' control after secondary operations involving levatorplasty, rerouting of the bowel and, in a few cases, transplantation of the gracilis muscle with or without the implantation of a radio-controlled muscle stimulator. However, as one would expect the results of secondary procedures do not compare with those of carrying out the puborectalis visualizing pullthrough in the first instance and only $26 \%$ approached normal.

The control of defaecation is a complex one with several factors. On the sensory side there is rectal sensation, anal canal sensation and perhaps stretch receptors in the puborectalis sling. On the motor side there is the striated muscle complex of puborecto-analis muscle and external sphincter. There is also the smooth muscle persistent tonus of the internal sphincter. In addition to this there are the anchorage factors of the perineal body and anococcygeal raphe which allow the muscles to lengthen and narrow the anal canal and maintain an anorectal angle as required. The resilience of the anal canal is also of importance and the lax attachment of the mucosa which allows the formation of a plug so that closure can be maintained with a reasonable amount of muscle action. In high anomalies one does one's best to minimize the interference with the sensory supply to the rectum and to preserve the striated muscle complex, but they have no internal sphincter and the anchorages of the anus are incomplete. It is my opinion that none of these obtain normal continence although one can, as described above, obtain a socially satisfactory continence through the cooperation of the child in learning to manage his incomplete mechanism. This contrasts with the results in the low abnormalities where one expects to get normal bowel control achieved in the normal time by routine baby management. Physiological tests including tests of the compliance of the anal canal ('yield pressure') have shown that in these in spite of the anatomical imperfection and the cut-back procedure there remains a normal internal sphincter tonus and reflex. It appears that the role of the internal sphincter in preventing soiling has been underestimated.

\section{References}

Bill, A.H. \& Johnson, R.J. (1958) Failure of migration of the rectal opening as the cause for most cases of imperforate anus. Surgery, Gynecology and Obstetrics, 106, 643.

Browne, D. (1951) Some congenital defects of the rectum, anus, vagina and urethra. Annals of the Royal College of Surgeons of England, 8, 173.

Browne, D. (1955) Congenital deformities of the anus and rectum. Archives of Diseases of Childhood, 30, 42.

GANS, S.L. \& Friedman, W.B. (1961) Some new concepts in the embryology, anatomy, physiology and surgical correction of imperforate anus. Western Journal of Surgery, Obstetrics and Gynecology, 69, 34.

KIESEWETTER, W.B. \& NiXON, H.H. (1967) Imperforate anus: its surgical anatomy. Journal of Pediatric Surgery, 3, 60.

KIESEWETTER, W.B. (1967) Imperforate anus II. The rationale and techniques of the sacro-abdominoperineal operation. Journal of Pediatric Surgery, 2, 106. 
Keith, Sir A. (1908) Some disorders of the hind end of the body. British Medical Journal, 2, 1736.

Kelly, J.H. (1969) The radiographic anatomy of the normal and abnormal pelvis. Journal of Pediatric Surgery, 4, 432.

LAwSON, J.O.N. (1969) The functional anatomy of the pelvic floor muscles and associated sphincters. In: Recent Advances in Paedriatric Surgery (Ed. by A. W. Wilkinson), 2nd edn. Churchill, London.

NixON, H.H. (1966) Colostomy: A simple technique and observations on indications. Zeitschrift fïr Kinderchirurgie und Grenzgebiete, 3, 98.

Santulli, T.V., Kiesewetter, W.B. \& Bill, A.H. JR (1970) Anorectal anomalies: A suggested international classification. Journal of Pediatric Surgery, 5, 281.
ScotT, J.E.S. (1959) The anatomy of the pelvic autonomic nervous system in cases of high imperforate anus. Surgery, $45,1013$.

ScotT, J.E.S. (1966) The microscopic anatomy of the terminal intestinal canal in ectopic vulval anus. Journal of Pediatric Surgery, 1, 441.

StePhens, F.D. (1963) In: Congenital Malformations of the Rectum, Anus and Genito-urinary Tracts. Livingstone, Edinburgh.

W ANGensteEN, O.H. \& RiCe, C.V. (1930) Imperforate anus. Annals of Surgery, 92, 77.

WiLSON, P. (1967) Anchoring mechanisms of the anorectal region. South African Medical Journal, 41, 1127. 